\title{
Evolution and performance of the ATLAS jet trigger
}

\author{
Mario Campanelli for the ATLAS collaboration \\ Department of Physics and Astronomy University College London \\ Gower Street WC1E 6BT London UK
}

\begin{abstract}
Jet-based trigger triggers allow the ATLAS experiment to perform an important first selection of physics events for use in studies of Standard Model processes and in searches for new physics at the LHC. The ATLAS ${ }^{1}$ jet trigger system has evolved substantially over the past few years of LHC operations, as experience accumulated with triggering in a high luminosity and high event pileup environment. In particular, the localised "region-of interest" (ROI) reconstruction strategy has been replaced by a full detector calorimeter reconstruction ("full scan") at the third trigger level, and by a full scan of the level-1 trigger input at level-2 for some specific trigger chains. Hadronic calibration and cleaning techniques are applied in order to provide improved performance and increased stability in high luminosity data taking conditions. In this presentation we describe the structure and performance of the ATLAS jet trigger during 2011 and 2012 data taking.

Keywords: Trigger; jets; Atlas
\end{abstract}

\section{Introduction}

Triggering on the Run 1 of the LHC was done under unprecedented conditions: the bunch-crossing spacing was $50 \mathrm{~ns}$ ( $25 \mathrm{~ns}$ for some special runs), with around 1000 bunches circulating in the detector per beam. This corresponds to a bunch-crossing rate of about $20 \mathrm{MHz}$, to be reduced to a maximal output rate available for physics of a few hundred Hz. This rejection factor of about $10^{5}$ was achieved by ATLAS using a 3-level trigger system exploiting the concept of Region Of Interest (ROI). That means that the Level-1 trigger system, based on custom electronics and in some cases detector hardware, will identify a region of the detector around which a high-energy calorimeter cluster, or a high-momentum muon track have been measured, and the following levels, Level-2 and Event Filter, will only focus on a part of the detector close to that produced particle. More details on the ATLAS trigger system can be found $\mathrm{in}^{2}$. 
Over the years, the accelerator luminosity has increased steadily, reaching about $8 \times 10^{33} \mathrm{~cm}^{2} \mathrm{~s}^{-1}$, corresponding to about 35 interactions per bunch crossing, close to the design value. Under these conditions, ATLAS trigger rates show signs of non-linear luminosity trend: the Level-1 minimum bias trigger rate flattened out, reaching the saturation value; the lowest-threshold jet trigger showed a quadratic behaviour and was switched off, while the Level-1 jet triggers with thresholds above $10 \mathrm{GeV}$ still had a quasi-linear behaviour, growing in rate by a factor of 5 for a luminosity increase of a factor of 4 . The detector occupancy in terms of size of the average event grew linearly with luminosity, therefore showing that saturation is not reached. The growth in event size shows that the DAQ system needs to cope with a growing data volume even at a fixed trigger-accept rate. In a typical run several trigger menus are deployed, with reduced thresholds as the instantaneous luminosity decreases, in order to maintain a constant trigger acceptance rate thus maximising the ATLAS physics intake.

\section{Level-1 jet triggers}

For the purpose of the first ATLAS trigger level (Level-1), the electromagnetic and hadronic calorimeters are segmented in trigger towers with minimal size $0.1 \times 0.1$ in the $\Delta \eta \times \Delta \phi$ plane. The Level- 1 electronics digitises only the faster part of the calorimeter electronics signal, providing a rough but quick estimate of the energy deposited in each tower. After pedestal subtraction a calibration, common between the $\mathrm{e} / \gamma, \tau$ and jet trigger selection paths (chains), is applied. The energy deposited in the electromagnetic part of the calorimeter is summed to that deposited in the hadronic part, so the final energy measurement is said to be at the electromagnetic scale. After that, a jet element is built from $2 \times 2$ trigger towers, and a jet algorithm based on square jet is used to find energy depositions above threshold. A region of interest is identified around local maxima of the energy deposition, and passed to the next level.

The efficiency of the Level-1 trigger chains is shown in figure 1, as a function of the offline jet transverse energy. The fact that thresholds are expressed at the electromagnetic scale explains why the mid-point of the turn-on curve happens at transverse energies higher than the trigger's nominal threshold. The Level-1 calorimeter resolution is responsible for the slow turn-on of these curves, especially at low energies.

In contrast to single-jet triggers the behaviour of multi-jet selections is rather non-linear: a factor of 10 rate increase was observed for a factor of 4 increase in luminosity. The black points in figure 2 illustrate the efficiency of 


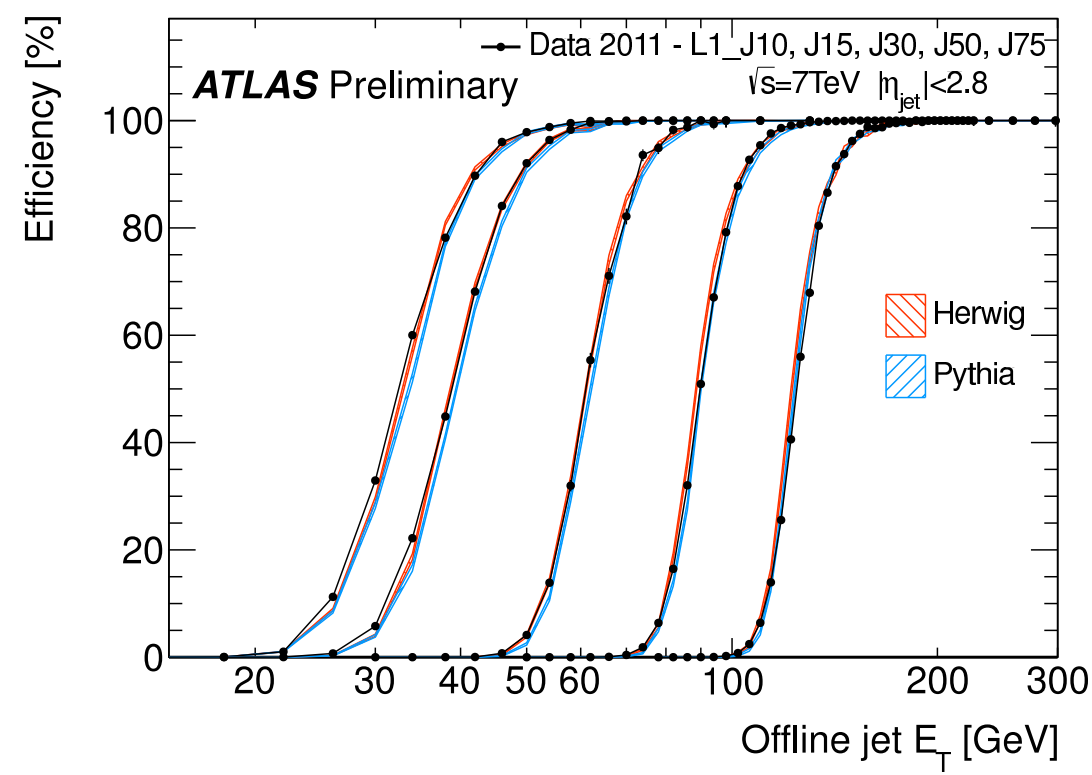

Fig. 1. The efficiency of Level-1 triggers in ATLAS. The thresholds are expressed at the electromagnetic scale, summing the energies deposited in the electromagnetic and hadronic parts of the calorimeter.

71 Level-1 multi-jet selections in ATLAS, where geometrical issues limit their

72 efficiency significantly below $100 \%$. This is due to the fact that complex

73 multi-jet configurations lose efficiency because of the "square" $\eta \times \phi$ jet

74 clusters, as opposed to the offline use of circular jet shapes, using the anti$75 k t^{3}$ algorithm.

To solve this problem, and add flexibility to the system, a new strategy has been devised. Bandwidth limitations prevent the ATLAS Level-2 triggers from reading the full calorimeter information; it is however possible to read out at Level-2 the energy deposition from all trigger towers from the Level-1 calorimeter system, and from them reconstruct jets at Level-2 using the same algorithm as used offline. The n-jets trigger efficiency can then be recovered selecting n-1 rectangular jets (reconstructed using square jets) 3 and request $\mathrm{n}$ jets at Level-2 from a full-scan of the Level-1 information. ${ }_{84}$ This approach almost fully recovers the efficiency in multijet configurations, 85 as shown by the red points in figure 2 .

${ }_{86}$ Further performance improvements from the use of full-scan of Level-1 ${ }_{87}$ information at Level-2 are an increased reconstruction efficiency for nearby 


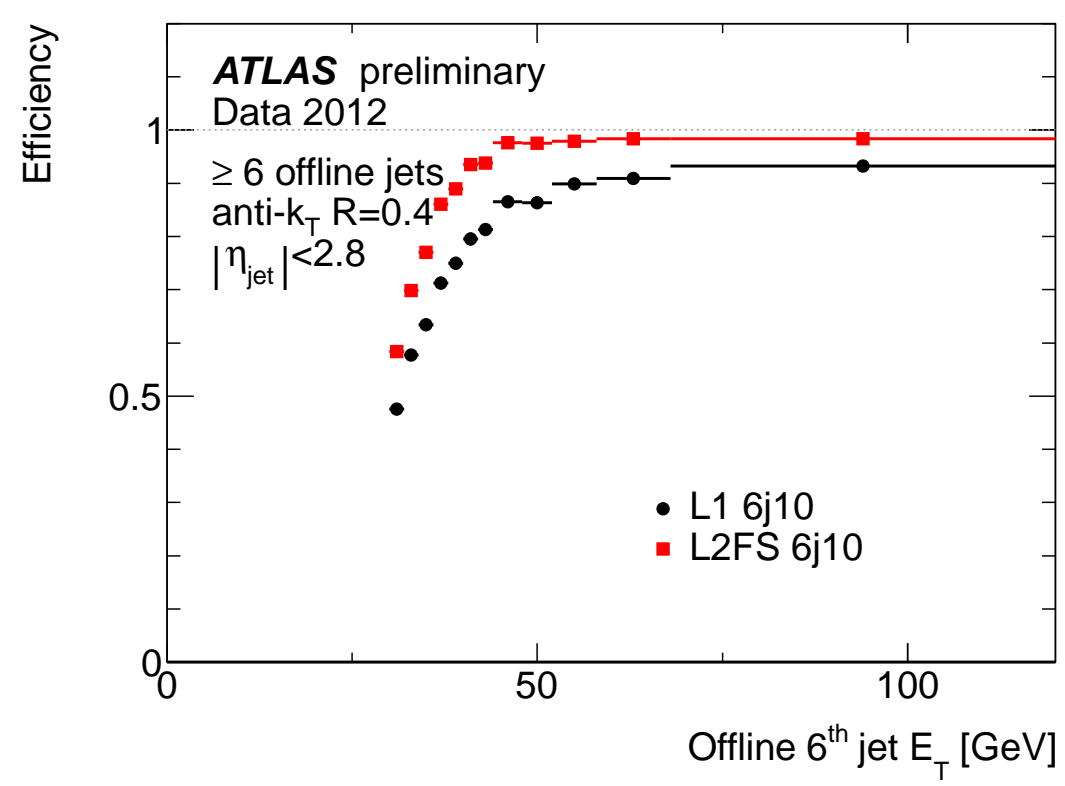

Fig. 2. The efficiency of Level-1 single-jet triggers in ATLAS, as measured from data and compared to full detector simulations, based on the HERWIG and PYTHIA Monte Carlo codes. The thresholds are expressed at the electromagnetic scale, summing the energies deposited in the electromagnetic and hadronic parts of the calorimeter.

jets, the possibility to apply an $\eta$-dependent jet intercalibration, with a sharpening of the turn-on of the efficiency curve, and a much better position resolution for forward jets: the Level-1 Calo trigger system has no segmentation in rapidity for jets with $\eta>3.2$, that are all reconstructed as having the average rapidity of the detector. Figure 3 shows how the resolution of Level-2 jets at large $\eta$ is also improved thanks to the ability to properly measure the jet rapidity from the level-1 calorimetric information.

\section{Event Filter triggers}

The Event Filter is the system taking the final decision about triggering the event. All events passing Level-2 are sent to the Event Filter, where the full calorimeter information is reconstructed and jets are created using the same algorithms as for offline analysis. The rates produced by the system were shown to behave in a similar way as those from Level-1: linear for single-jet chains, increasing as much as twice faster than the luminosity for 


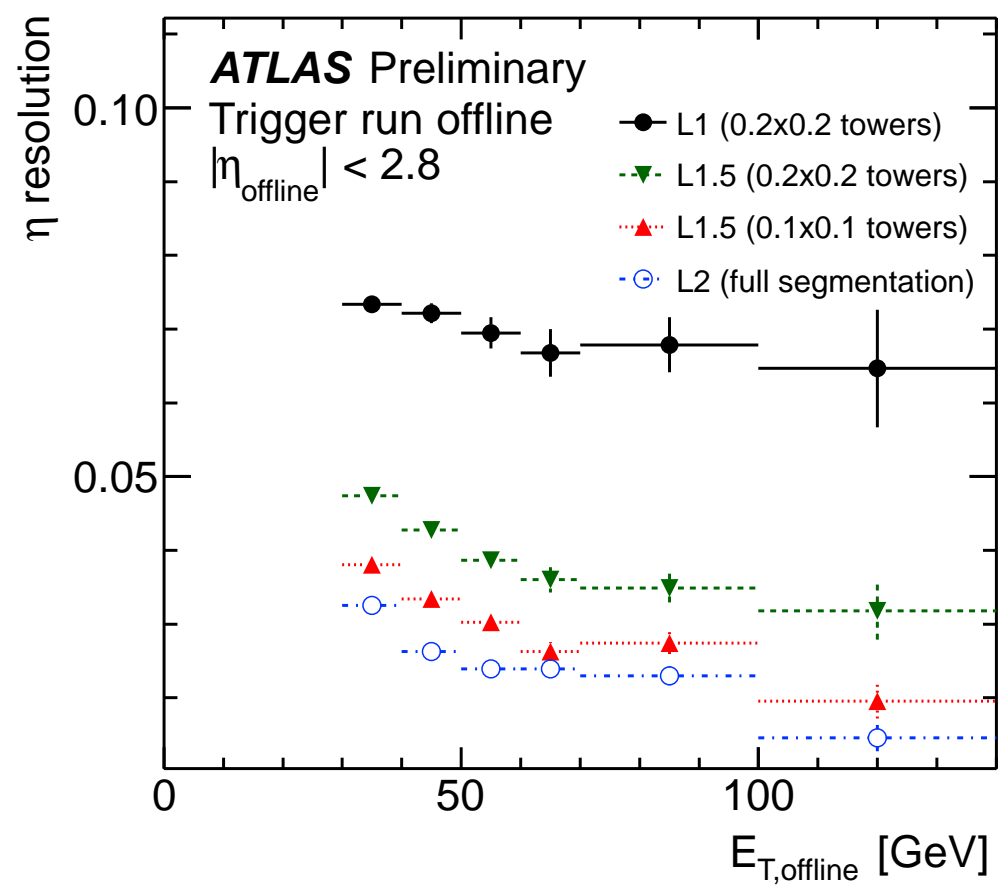

Fig. 3. The rapidity resolution for the hardware Level-1 system, for reconstructing Level-1 Calo towers at Level-2 (indicated as L1.5) using different granularity, and from the full Level-2 segmentation (indicated as L2)

the multi-jet ones. The thresholds for EF trigger chains have been designed to have similar full-efficiency points as the Level-1 triggers, to optimise bandwidth. Efficiency curves like the ones from figure 4 can be measured on data thanks to a small fraction of randomly selected (thus completely unbiased by trigger selections) events that bypass all Level-1 and Level-2 requirements.

This idea allows being fully efficient for jets that are not taken by even the lowest threshold of the Level-1 jet trigger system.

Figure 4 shows a significant discrepancy between these efficiency curves and those expected from the Monte Carlo simulation. Also the jet resolution is badly described, with data having a better resolution through the whole $p_{T}$ range, as shown by figure 5 . 


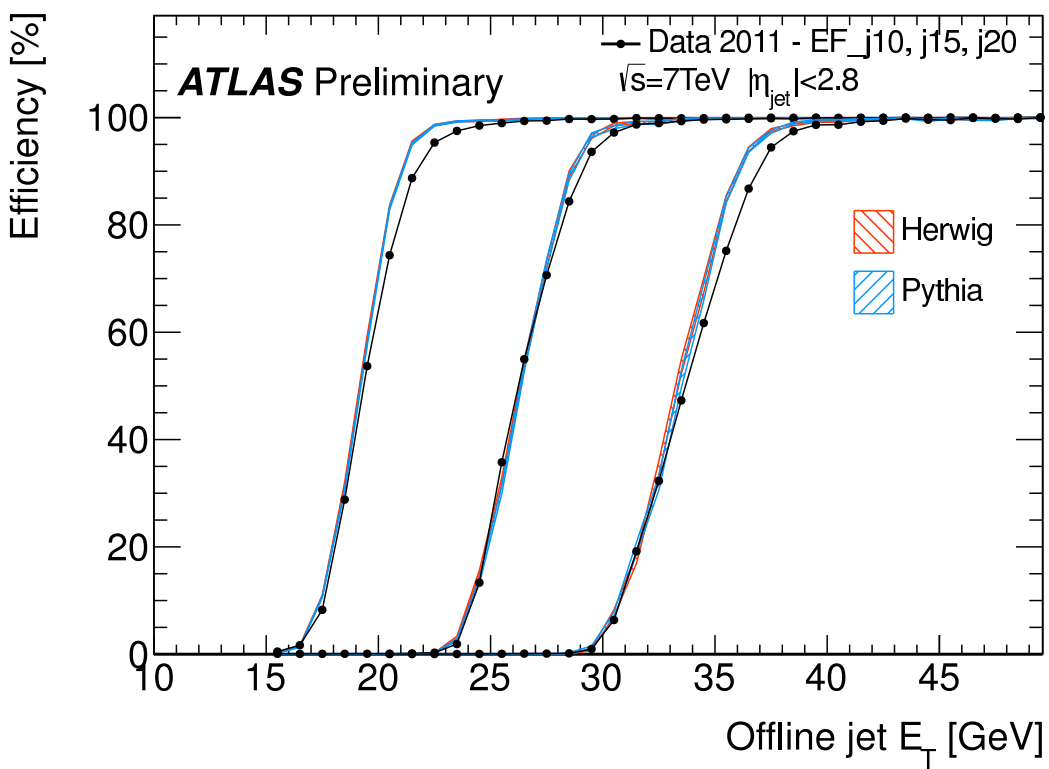

Fig. 4. The Event Filter efficiencies for the low transverse momentum jet trigger thresholds, comparing data (black circles) and simulation (blue and red continuous lines).

\section{Next steps and conclusions}

After the current shutdown (2013-2015), the Level-2 and Event Filter codes will be run on the same computer node for all ATLAS triggers, and the jet trigger system will use this opportunity to merge the two into a single piece of High-Level Trigger code. For multi-jets, the Level-1 based full-scan information will still be used before the final selection to reduce the number of events. A dedicated hardware will provide the High Level Trigger with charged track measurements, allowing event-by-event pileup subtraction, and the cut on relative jet angles will be possible using a new topological module to be operational at Level-1. In conclusion, the ATLAS jet trigger system has overcome the limitations of the Region Of Interest approach, providing full-scan of the calorimeter both at Level-2 (using Level-1 information) and at Event Filter. These new approaches allowed full efficiency for multijet topologies, better response in the forward region, the extension of triggering capabilities to very low transverse momenta and better overall response. The foreseen integration of Level- 2 and Event Filter will produce a smoother system, where HLT jets will be almost identical to the offline 


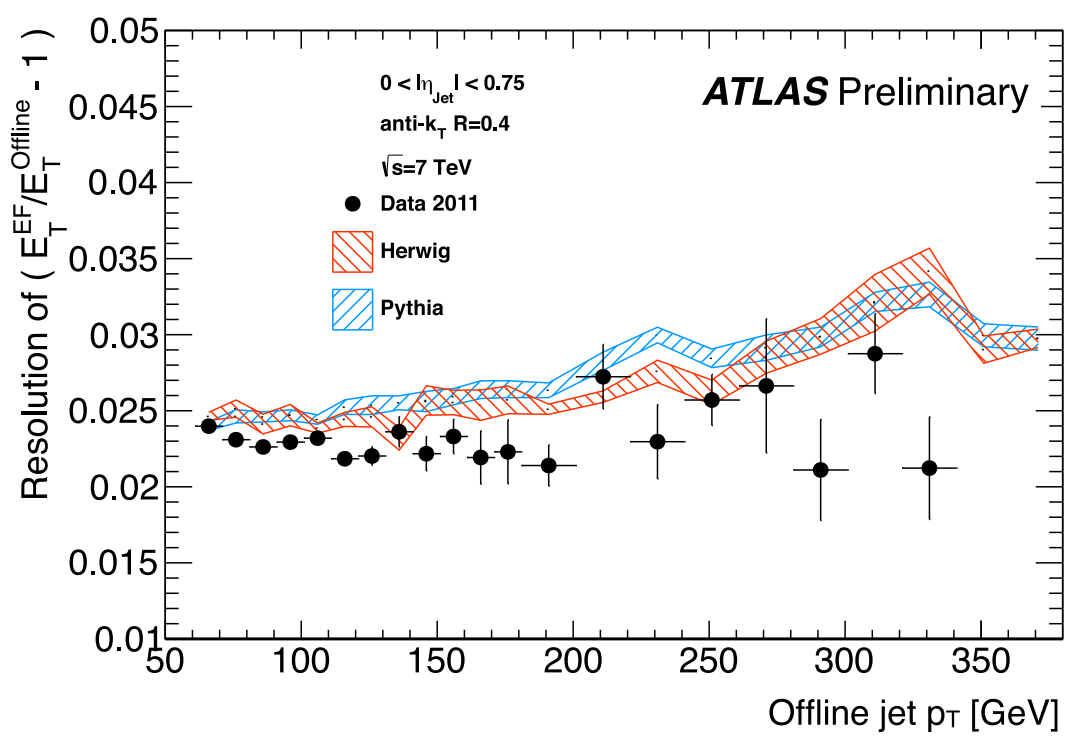

Fig. 5. A comparison between Event Filter resolutions in data and for the Pythia and Herwig Monte Carlo codes.

ones.

\section{References}

1. ATLAS Collaboration, The ATLAS Experiment at the CERN Large Hadron Collider, JINST 3 (2008) S08003.

2. G. Aad et al. [ATLAS Collaboration], "Performance of the ATLAS Trigger System in 2010," Eur. Phys. J. C 72, 1849 (2012) [arXiv:1110.1530 [hep-ex]].

3. M. Cacciari, G. P. Salam and G. Soyez, "The Anti-k(t) jet clustering algorithm," JHEP 0804, 063 (2008) [arXiv:0802.1189 [hep-ph]]. 